\title{
THE INTEGRABILITY PROBLEM FOR LIE EQUATIONS
}

\author{
BY HUBERT GOLDSCHMIDT ${ }^{\prime}$
}

The study of pseudogroups and geometric structures on manifolds associated to them was initiated by Sophus Lie and Élie Cartan. In comparing two such structures, Cartan was led to formulate the equivalence problem and solved it in the analytic case using the Cartan-Kähler theorem (see [2] and [3]). In the early 1950's, Spencer elaborated a program to study structures on manifolds, in particular complex structures, and their deformations in terms of partial differential equations. He set up the integrability problem for Lie pseudogroups and introduced partial differential equations which are equivalent to those solved by Cartan when all the data are real-analytic. In the case of complex structures, this partial differential equation expresses the fact that an almost-complex structure is actually a complex structure. In 1957, Newlander and Nirenberg showed that every formally integrable almost-complex structure is integrable, and is in fact a complex structure. Spencer conjectured that the corresponding result would hold for any elliptic Lie pseudogroup; this was proved by Malgrange [19] under the additional assumption that the pseudogroup is analytic. However in 1967, Guillemin and Sternberg [16] gave an example, based on H. Lewy's counterexample to the local solvability of partial differential equations, which shows that the integrability problem is not always solvable. On the other hand, Guillemin and Pollack [21] and Buttin-Molino ([1] and [20]) studied the integrability problem for flat Lie pseudogroups on $\mathbf{R}^{n}$ and obtained partial results.

In this paper, we give an outline of our proof of the solvability of the integrability problem for all Lie pseudogroups acting on $\mathbf{R}^{n}$ which contain the translations (Theorem 10), a fortiori for all flat pseudogroups. It is a summary of joint work with D. C. Spencer ([12] and [13]) and follows to a large extent Guillemin's program (described in the introduction of [14]) for solving the integrability problem for flat pseudogroups by means of Galois theory type methods similar to those introduced by Sophus Lie in his work on partial differential equations. Guillemin's Jordan-Hölder decomposition for transitive Lie algebras (see [14]) is the required algebraic tool, together with the classification of the simple transitive pseudogroups and the results of Guillemin [14] and Conn [5] on the structure of nonabelian minimal closed ideals of real transitive Lie algebras. We also need the result in several special cases, which is given by the Frobenius, Darboux and Newlander-Nirenberg

An Invited Address delivered at the St. Louis, Missouri meeting on January 27, 1977; received by the editors October 25, 1977.

AMS (MOS) subject classifications (1970). Primary 58H05, 22E65, 58G99, 35N10, $53 \mathrm{Cl0}$.

${ }^{1}$ This work was supported in part by National Science Foundation Grant MCS 76-23465. 
theorems, as well as the Ehrenpreis-Malgrange theorem which asserts that over-determined constant coefficient linear differential operators are locally solvable. Moreover, we give a unified treatment in terms of pseudo-complex structures (also called $C R$-structures) of the counterexamples of GuilleminSternberg [16] and Conn [4] to the solvability of the integrability problem.

1. The integrability problem for $G$-structures. We begin by considering the following problems:

(a) Let $X$ be the Riemannian manifold $\mathbf{R}^{n}$ with the Euclidean metric and $X^{\prime}$ be an $n$-dimensional Riemannian manifold. Find local isometries $f$ : $X \rightarrow X^{\prime}$.

(b) Let $X$ be the symplectic manifold $\mathbf{R}^{2 n}$, with coordinates $\left(x^{1}, \ldots, x^{n}, y^{1}, \ldots, y^{n}\right)$ and the standard symplectic form $\sigma=d x^{1} \wedge d y^{1}$ $+\cdots+d x^{n} \wedge d y^{n}$. If $X^{\prime}$ is a $2 n$-dimensional symplectic manifold, that is, a manifold endowed with a 2-form $\sigma^{\prime}$ of maximal rank, find local diffeomorphisms $f: X \rightarrow X^{\prime}$ such that $f^{*} \sigma^{\prime}=\sigma$.

(c) Let $X^{\prime}$ be a $2 n$-dimensional almost-complex manifold. Find local diffeomorphisms $f: \mathbf{C}^{n} \rightarrow X^{\prime}$ such that the almost-complex structure on $X^{\prime}$ is that induced by $f$ from that of $\mathbf{C}^{n}$.

(d) Let $X^{\prime}$ be an $n$-dimensional manifold together with a distribution $V^{\prime}$ of rank $k$, that is, a subbundle of the tangent bundle of $X^{\prime}$ of rank $k$. Let $X=\mathbf{R}^{n}$ and consider the projection $\rho: X \rightarrow \mathbf{R}^{n-k}$ sending $\left(x^{1}, \ldots, x^{n}\right)$ into $\left(x^{k+1}, \ldots, x^{n}\right)$. If $V$ is the bundle of vectors tangent to the fibers of $\rho$, find local diffeomorphisms $f: X \rightarrow X^{\prime}$ such that $f_{*}\left(V_{x}\right)=V_{f(x)}^{\prime}$, for all $x$ belonging to the domain of $f$.

In all these problems, in order for a local diffeomorphism $f: X \rightarrow X^{\prime}$ defined on a neighborhood of $x \in X$ to satisfy the corresponding condition, the Taylor series of $f$ at $x$ must verify an algebraic relation. The existence of such Taylor series imposes a formal integrability condition on the structure on $X^{\prime}$. In each of the above problems, this necessary condition for the existence of the diffeomorphisms $f$ is in fact also sufficient according to various well-known results. We now examine each problem separately.

(a) The formal integrability condition is equivalent to the vanishing of the curvature of the Riemannian manifold $X^{\prime}$. If this curvature vanishes, then existence theorems for differential equations give us the desired diffeomorphisms.

(b) The necessary condition is $d \sigma^{\prime}=0$. If $\sigma^{\prime}$ is closed, then the existence of the diffeomorphisms is a consequence of Darboux's theorem.

(c) The condition in this case is the formal integrability of the almost-complex structure on $X^{\prime}$. The Newlander-Nirenberg theorem asserts that such a structure is in fact a complex structure.

(d) That the space of sections of $V^{\prime}$ be stable under the Lie bracket is the formal integrability condition for $V^{\prime}$. Frobenius' theorem then says that, under that assumption, $V^{\prime}$ is a foliation and thus gives us the desired diffeomorphisms.

We proceed to give a unifying viewpoint for these various problems. First, we define the notion of a pseudogroup.

Definition. A collection $\Gamma$ of local diffeomorphisms of a manifold $X$ is a 
pseudogroup if the following holds:

(i) if $f_{1}: U \stackrel{\sim}{\rightarrow} V$ and $f_{2}: V \stackrel{\sim}{\rightarrow} W$ belong to $\Gamma$, then $f_{2} \circ f_{1}$ belongs to $\Gamma$;

(ii) if $f: U \stackrel{\sim}{\rightarrow} V$ belongs to $\Gamma$, so does $f^{-1}: V \stackrel{\sim}{\rightarrow} U$;

(iii) the identity mapping of $X$ belongs to $\Gamma$;

(iv) if $U=\cup_{\alpha \in A} U_{\alpha}$ and $f: U \stackrel{\sim}{\rightarrow} V$ is a local diffeomorphism, then $f \in \Gamma$ if and only if $f_{\mid U_{\alpha}} \in \Gamma$ for all $\alpha \in A$.

For a pseudogroup to be a Lie pseudogroup, the diffeomorphisms of $\Gamma$ must satisfy a partial differential equation.

Let $G \subset \mathrm{Gl}(n, \mathbf{R})$ be a Lie subgroup. Consider the set $\Gamma_{G}$ of all local diffeomorphisms $f=\left(f^{1}, \ldots, f^{n}\right)$ of $\mathbf{R}^{n}$ satisfying

$$
\left(\frac{\partial f^{j}}{\partial x^{i}}(x)\right) \in G
$$

for all $x$ belonging to the domain of $f$; it is a Lie pseudogroup.

If $G=O(n)$, then $\Gamma_{G}$ is the set of all Euclidean motions, that is, of local isometries of the Riemannian manifold $X$ of problem (a).

If $G=\operatorname{Sp}(n, \mathbf{R})$, considered as a subgroup of $\operatorname{Gl}(2 n, \mathbf{R})$, then $\Gamma_{G}$ is the set of all local symplectic diffeomorphisms of $\mathbf{R}^{2 n}$, i.e., diffeomorphisms preserving the 2-form $\sigma$ of problem (b).

If $G=\mathrm{Gl}(n, \mathrm{C})$, considered as a subgroup of $\mathrm{Gl}(2 n, \mathrm{R})$, then $\Gamma_{G}$ is the set of all biholomorphic mappings of $\mathbf{C}^{n}$ and therefore consists of all diffeomorphisms preserving the almost-complex structure of $\mathbf{C}^{n}$.

If $G$ is the subgroup $\mathrm{Gl}(n, k ; \mathbf{R})$ of $\mathrm{Gl}(n, \mathbf{R})$ consisting of all matrices leaving invariant the $k$-dimensional subspace of $\mathbf{R}^{n}$ defined by $x_{k+1}$ $=\cdots=x_{n}=0$, then $\Gamma_{G}$ is the pseudogroup of local diffeomorphisms preserving the foliation of $\mathbf{R}^{n}$ whose leaves are the fibers of the mapping $\rho$ of problem (d).

We formulate the integrability problem for the pseudogroup $\Gamma_{G}$. A $G$-structure $\sigma^{\prime}$ on a manifold $X^{\prime}$ of dimension $n$ is a reduction of the principal bundle of the tangent bundle of $X^{\prime}$ to the group $G$. Such a $G$-structure $\sigma^{\prime}$ on $X^{\prime}$ is equivalent to the following data:

(i) an open covering $\left\{U_{\alpha}\right\}_{\alpha \in A}$ of $X^{\prime}$;

(ii) on each open set $U_{\alpha}$ (with $\alpha \in A$ ), a coframe $\omega_{\alpha}=\left(\omega_{\alpha}^{1}, \ldots, \omega_{\alpha}^{n}\right)$ such that on $U_{\alpha} \cap U_{\beta}$

$$
\omega_{\alpha}=g_{\alpha \beta} \cdot \omega_{\beta},
$$

where $g_{\alpha \beta}: U_{\alpha} \cap U_{\beta} \rightarrow G$.

We say that a coframe $\omega$ on an open subset $V$ of $X^{\prime}$ belongs to the $G$-structure $\sigma^{\prime}$ if

$$
\omega=g_{\alpha} \cdot \omega_{\alpha} \text { on } V \cap U_{\alpha},
$$

where $g_{\alpha}: V \cap U_{\alpha} \rightarrow G$.

On $\mathbf{R}^{n}$, we have the standard $G$-structure consisting of the coframe $\sigma=$ $\left(d x^{1}, \ldots, d x^{n}\right)$. A $G$-structure $\sigma^{\prime}$ on $X^{\prime}$ is said to be integrable if, for every $x_{0}^{\prime} \in X^{\prime}$, there is a local diffeomorphism $f: U \rightarrow X^{\prime}$ defined on a neighborhood $U$ of 0 in $\mathbf{R}^{n}$ such that $f(0)=x_{0}^{\prime}$ and a mapping $g: U \rightarrow G$ satisfying

$$
f^{*} \omega=g \cdot \sigma,
$$


where $\omega$ is a coframe belonging to the $G$-structure $\sigma^{\prime}$ on $V=f(U)$. The equation (1) is a nonlinear partial differential equation. If $g(x)$ is the matrix $\left(g_{i}^{j}(x)\right)$, for $x \in U$, in terms of coordinates $\left(y^{1}, \ldots, y^{n}\right)$ on $V$, we may write

$$
\omega^{j}=\sum_{k=1}^{n} \omega_{k}^{j} d y^{k}
$$

and $f=\left(f^{1}, \ldots, f^{n}\right)$ and equation (1) can be written as the equalities

$$
\sum_{k=1}^{n} \frac{\partial f^{k}}{\partial x^{i}}\left(\omega_{k}^{j} \circ f\right)=g_{i}^{j}, \quad i, j=1, \ldots, n,
$$

on $U$. Let $\varphi=f^{-1}$ and $h: V \rightarrow G$ be the mapping given by $h(y)=$ $g(\varphi(y))^{-1}$, for $y \in V$; then $\varphi\left(x_{0}^{\prime}\right)=0$ and equation (1) is equivalent to

$$
d \varphi=h \cdot \omega
$$

on $V$. If $\varphi=\left(\varphi^{1}, \ldots, \varphi^{n}\right)$ and $h(y)$ is the matrix $\left(h_{i}^{j}(y)\right)$, for $y \in V$, then (2) can be written as the equalities

$$
\frac{\partial \varphi^{j}}{\partial y^{k}}=\sum_{i=1}^{n} h_{i}^{j} \omega_{k}^{i}
$$

on $V$.

If $f: U \rightarrow X^{\prime}$ is a local diffeomorphism defined on an open subset $U$ of $\mathbf{R}^{n}$ satisfying equation (1) for some $G$-valued function $g$ on $U$ and some coframe $\omega$ belonging to the $G$-structure $\sigma^{\prime}$ on $V=f(U)$, and if $\varphi: V \rightarrow \mathbf{R}^{n}$ is a local diffeomorphism satisfying (2) for some $G$-valued function $h$ on $V$, then it is easily seen that $\varphi \circ f$ belongs to the pseudogroup $\Gamma_{G}$. Thus the integrability of a $G$-structure implies the existence of coordinates for the structure, that is, of an atlas whose coordinate transformations belong to $\Gamma_{G}$.

The problems (a)-(d) considered at the beginning of this section are the integrability problems for $G$-structures, with $G=O(n), \mathrm{Sp}(n, \mathbf{R}), \mathrm{Gl}(n, \mathrm{C})$ and $\mathrm{Gl}(n, k ; \mathbf{R})$ respectively. As in these problems, for a $G$-structure $\sigma^{\prime}$ on $X^{\prime}$ to be integrable, it must necessarily satisfy a formal integrability condition: for each $x_{0}^{\prime} \in X^{\prime}$, there is a coframe $\omega$ belonging to the $G$-structure $\sigma^{\prime}$ on a neighborhood of $x_{0}^{\prime}$, a local diffeomorphism $f: \mathbf{R}^{n} \rightarrow X^{\prime}$ and a mapping $g$ : $\mathbf{R}^{n} \rightarrow G$ defined on a neighborhood of 0 in $\mathbf{R}^{n}$ such that $f(0)=x_{0}^{\prime}$ and (1) holds to infinite order at 0 . The integrability problem for $G$-structures, or for the pseudogroup $\Gamma_{G}$, is to show that every formally integrable $G$-structure is integrable. As we have already mentioned, the problem is solved when $G$ is one of the four groups considered above. In the remainder of this paper, we present a solution of a generalization of this integrability problem.

2. The integrability problem for pseudogroups. Let $\Gamma$ be a Lie pseudogroup acting on an $n$-dimensional manifold $X$. It is easily seen that the sheaf of infinitesimal transformations of $\Gamma$, that is, of vector fields $\theta$ on $X$ for which $\exp t \theta$ belongs to $\Gamma$ for all small $t$, is stable under the Lie bracket. This sheaf consists of solutions of a linear partial differential equation, which is called a Lie equation.

If $G \subset \mathrm{Gl}(n, \mathbf{R})$ is a Lie subgroup and $g \subset g l(n, \mathbf{R})$ is its Lie algebra, the sheaf of infinitesimal transformations of $\Gamma_{G}$ consists of those vector fields 
$\theta=\sum_{j=1}^{n} \theta^{j} \partial / \partial x^{j}$ defined on open subsets $U$ of $\mathbf{R}^{n}$ satisfying the linear partial differential equation

$$
\left(\frac{\partial \theta^{j}}{\partial x^{i}}(x)\right) \in \mathfrak{g}
$$

for all $x \in U$.

We now consider the class of linear partial differential equations which the infinitesimal transformations of a pseudogroup satisfy. We begin by recalling various facts from the formal theory of linear differential equations (see [6] and [7]). If $E$ is a bundle over $X$, we denote by $\mathcal{E}$ the sheaf of sections of $E$ and by $\varepsilon_{x}$ its stalk at $x \in X$. If $E$ is a vector bundle over $X$, we denote by $J_{k}(E)$ the vector bundle of $k$-jets of sections of $E$ and by $\pi_{k}: J_{k+l}(E) \rightarrow$ $J_{k}(E)$ the natural projection.

A subbundle $R_{k} \subset J_{k}(E)$ is a linear differential equation of order $k$ in $E$. A section $u$ of $E$ is a solution of $R_{k}$ if the $k$-jet $j_{k}(s)$ of $s$ is a section of $R_{k}$. If $F$ is another vector bundle over $X$, a linear differential operator $P: \mathcal{E} \rightarrow \mathscr{F}$ of order $k$ is said to be associated to $R_{k}$ if $R_{k}$ is the kernel of the morphism of vector bundles $p_{0}(P): J_{k}(E) \rightarrow F$ induced by $P$. In fact, $s \in \mathcal{E}$ is a solution of $R_{k}$ if and only if $P s=0$. It is clear that there always exists such a differential operator $P$ associated to $R_{k}$.

For $l>0$, we associate a subbundle $R_{k+l} \subset J_{k+l}(E)$ with varying fiber in the following way. Choose a differential operator $P: \mathcal{E} \rightarrow \mathscr{F}$ associated to $R_{k}$; then $R_{k+l}$ is the kernel of the morphism of vector bundles

$$
p_{l}(P): J_{k+l}(E) \rightarrow J_{l}(F)
$$

which sends $j_{k+l}(s)(x)$ into $j_{l}(P s)(x)$, if $s$ is a section of $E$ over a neighborhood of $x \in X$. Moreover, $R_{k+l}$ depends only on $R_{k}$ and $R_{k+l, x}$ is the space of solutions of $P s=0$ of order $k+l$ at $x$. Hence $\pi_{k+l}$ maps $R_{k+l+1}$ into $R_{k+l}$, so we can set $R_{\infty}=\operatorname{proj} \lim R_{k+l}$; this is the space of formal solutions of the equation $P s=0$.

Definition. We say that a differential equation $R_{k} \subset J_{k}(E)$ is formally integrable if $R_{k+l}$ is a vector bundle and if the projection $\pi_{k+l}: R_{k+l+1} \rightarrow$ $R_{k+l}$ is surjective, for all $l \geqslant 0$.

We denote by $T=T(X)$ the tangent bundle of $X$. In [22], Spencer introduced a first-order linear differential operator

$$
D: \wedge \mathcal{T}^{*} \otimes J_{m}(\mathcal{E}) \rightarrow \wedge \mathcal{T}^{*} \otimes J_{m-1}(\mathcal{E})
$$

which is uniquely characterized by the conditions:

$$
\begin{aligned}
& D\left(j_{m}(s)\right)=0, \text { for all } s \in \mathcal{E}, \\
& D(\omega \wedge u)=d \omega \wedge \pi_{m-1} u+(-1)^{j} \omega \wedge D u,
\end{aligned}
$$

for all $\omega \in \wedge^{j} \mathcal{T}^{*}, u \in \wedge \mathcal{T}^{*} \otimes J_{m}(\mathcal{T})$. It is easily seen that $D^{2}=0$.

Let $R_{k} \subset J_{k}(E)$ be a formally integrable differential equation of order $k$ in $E$. If we write $R_{m}=J_{m}(T)$ for $m<k$, this operator $D$ gives us by restriction the linear Spencer complex

$$
0 \rightarrow \Re_{m} \stackrel{D}{\rightarrow} \mathcal{J}^{*} \otimes \Re_{m-1} \stackrel{D}{\rightarrow} \Lambda^{2} \mathcal{T}^{*} \otimes \Re_{m-2} \rightarrow \cdots \rightarrow \Lambda^{n} \mathcal{T}^{*} \otimes \Re_{m-n} \rightarrow 0 . \text { (3) }
$$


Let $P: \mathcal{E} \rightarrow \mathscr{F}$ be a differential operator of order $k$ associated to $R_{k}$. According to [6] there exist a vector bundle $G$ and a differential operator $Q$ : $\mathscr{F} \rightarrow \mathcal{G}$ of order $l$ associated to a formally integrable differential equation of order $l$ in $G$ such that

$$
\mathcal{E} \stackrel{P}{\rightarrow} \mathscr{F} \stackrel{Q}{\rightarrow} \mathcal{G}
$$

is a formally exact complex in the sense that the sequences

$$
J_{k+l+m}(E) \stackrel{p_{l+m}(P)}{\rightarrow} J_{l+m}(F) \stackrel{p_{m}(Q)}{\rightarrow} J_{m}(G)
$$

are exact for $m \geqslant 0$. Moreover the cohomology of the complex (4) depends only on $R_{k}$ and is isomorphic to the cohomology $H^{1}\left(R_{k}\right)_{m-1}$ of the sequence (3) at $\mathfrak{T}^{*} \otimes \Re_{m-1}$, for $m \geqslant k+l$. The operator $Q$ is the compatibility condition for $\boldsymbol{P}$ and expresses all the formal obstructions to solving the inhomogeneous equation $P s=f$ with $f \in \mathcal{F}$. Therefore the cohomology $H^{1}\left(R_{k}\right)_{m-1}$ is independent of $m$ for $m \geqslant k+l$, and is the obstruction to the local solvability of the differential operator $P$; it is called the first (linear) Spencer cohomology group of $R_{k}$ (see [7]).

We shall impose the condition on a differential equation $R_{k} \subset J_{k}(T)$ that, if vector fields $\xi, \eta$ are solutions of $R_{k}$, then the bracket $[\xi, \eta]$ also be a solution of $R_{k}$. If we set

$$
\left[j_{k}(\xi)(x), j_{k}(\eta)(x)\right]=j_{k-1}[\xi, \eta](x),
$$

where $\xi, \eta$ are vector fields defined on a neighborhood of $x \in X$, we obtain a bracket

$$
J_{k}(T)_{x} \otimes J_{k}(T)_{x} \rightarrow J_{k-1}(T)_{x}
$$

making $J_{\infty}(T)_{x}=$ proj $\lim J_{m}(T)_{x}$ into a Lie algebra.

Definition. A formally integrable equation $R_{k} \subset J_{k}(T)$ of order $k$ in $T$ is said to be a Lie equation if $\left[R_{k+1}, R_{k+1}\right] \subset R_{k}$. A Lie equation is formally transitive if $\pi_{0}: R_{k} \rightarrow J_{0}(T)$ is surjective.

The infinitesimal transformations of a Lie pseudogroup are the solutions of a Lie equation. If the pseudogroup is transitive, then this Lie equation is formally transitive. The theory of Lie equations was elaborated by Spencer, Malgrange and Kumpera (see [22], [19] and [17]).

If $R_{k} \subset J_{k}(T)$ is a Lie equation, then so is $R_{k+l}$ for all $l \geqslant 0$ and, for $x \in X$, the subspace $R_{\infty, x}=$ proj $\lim R_{k+l, x}$ of $J_{\infty}(T)_{x}$ is a subalgebra. We now construct a nonlinear analogue of the complex (3). We consider the groupoid $Q_{m}$ of $m$-jets of local diffeomorphisms of $X$ as a bundle over $X$ via the projection sending the $m$-jet $j_{m}(f)(x)$ of a diffeomorphism $f$ at $x \in X$ into $x$. In $\$ 5$ of [12], We define the Cartan fundamental form $\omega$ on $Q_{m+1}$

$$
\omega: T\left(Q_{m+1}\right) \rightarrow J_{m}(T)
$$

with values in $J_{m}(T)$; its restrictions to the fibers of $Q_{m+1}$ over $X$ are the classical fundamental forms of Cartan. We obtain a nonlinear differential operator

$$
\text { Q }: 2_{m+1} \rightarrow \mathscr{T}^{*} \otimes J_{m}(\mathfrak{T})
$$

sending a section $F$ of $Q_{m+1}$ into the $J_{m}(T)$-valued 1-form $\mathscr{D} F=F^{*} \omega$ on $X$ 
defined by

$$
\left\langle\xi, F^{*} \omega\right\rangle=\left\langle F_{*} \xi, \omega\right\rangle, \text { for } \xi \in T .
$$

The fundamental form $\omega$ satisfies a Cartan structure equation of the form

$$
D \omega-\frac{1}{2}[\omega, \omega]=0 \text {, }
$$

whose bracket is induced by (5). Consider the bracket

$$
\left(\wedge^{i} T^{*} \otimes R_{m}\right) \otimes\left(\wedge^{j} T^{*} \otimes R_{m}\right) \rightarrow \wedge^{i+j} T^{*} \otimes R_{m-1}
$$

defined by the formula

$$
[\alpha \otimes \xi, \beta \otimes \eta]=(\alpha \wedge \beta) \otimes[\xi, \eta]
$$

where $\alpha \in \wedge^{i} T^{*}, \beta \in \wedge^{j} T^{*}, \xi, \eta \in R_{m}$. If

$$
\mathscr{D}_{1}: \mathscr{T}^{*} \otimes J_{m}(\mathfrak{T}) \rightarrow \wedge^{2} \mathfrak{T}^{*} \otimes J_{m-1}(\mathfrak{T})
$$

is the operator given by

$$
\mathscr{D}_{1} u=D u-\frac{1}{2}[u, u]
$$

for $u \in \mathscr{T}^{*} \otimes J_{m}(\mathfrak{T})$, we derive from the structure equation for $\omega$ the identity

$$
\mathscr{Q}_{1} \mathscr{Q} F=0
$$

for $F \in \mathcal{2}_{m+1}$ (see [12, §5]). The following Spencer complex

$$
\mathcal{2}_{m+1} \stackrel{\mathscr{D}}{\rightarrow} \mathcal{T}^{*} \otimes J_{m}(\mathfrak{T}) \stackrel{\mathscr{D}_{1}}{\rightarrow} \wedge^{2} \mathscr{T} * \otimes J_{m-1}(\mathfrak{T})
$$

is a nonlinear analogue of the initial portion of (3), with $R_{k}=J_{k}(T)$.

Let $\Gamma$ be a Lie pseudogroup acting on $X$. Its infinitesimal transformations are the solutions of a Lie equation $R_{k} \subset J_{k}(T)$ of order $k$; let $l$ be the order of the differential operator $Q$ obtained above from $R_{k}$ and a differential operator $P$ associated to $R_{k}$. The set of all $m$-jets of diffeomorphisms belonging to $\Gamma$ is a subgroupoid and subbundle $P_{m}$ of $Q_{m}$, for $m \geqslant k$; the $m$-jet $I_{m}(x)$ of the identity mapping of $X$ at $x$ belongs to $P_{m}$ for $m \geqslant k$. In fact, $P_{k}$ is an integrable and formally integrable finite form of the Lie equation $R_{k}$ in the sense of [12], whose $r$ th prolongation is $P_{k+r}$. Finally, the restriction of the fundamental form $\omega$ to $P_{m+1}$ is $R_{m}$-valued for $m \geqslant k$ and so we obtain the subcomplex of (6)

$$
\mathcal{P}_{m+1} \stackrel{\mathscr{D}}{\rightarrow} \mathscr{T} * \otimes \mathcal{R}_{m} \stackrel{\mathscr{D}_{1}}{\rightarrow} \wedge^{2} \mathcal{T}^{*} \otimes \mathcal{R}_{m-1} .
$$

We are now able to state the integrability problem for the pseudogroup $\Gamma$ in terms of the sequence (7):

Let $m \geqslant k+l-1$ and $x \in X$. Given $u \in\left(\mathcal{T}^{*} \otimes \Re_{m}\right)_{x}$, with $u(x)=0$ and $\mathscr{D}_{1} u=0$, find $F \in \mathscr{P}_{m+1, x}$ satisfying $F(x)=I_{m+1}(x)$ and

$$
\mathscr{Q} F=u \text {. }
$$

This problem for the pseudogroup $\Gamma_{G}$ acting on $\mathbf{R}^{n}$ is equivalent to the integrability problem posed in $\S 1$. For an arbitrary pseudogroup $\Gamma$, the solvability of this problem is in fact independent of the choice of the integer $m \geqslant k+l-1$. The element $u$ corresponds to a germ of a "structure associated to $\Gamma$ " and the equation (8) expresses the integrability of this 
structure. The relation $\mathscr{D}_{1} u=0$ is the formal integrability condition for this structure and for the local solvability of (8).

To any Lie equation $R_{k} \subset J_{k}(T)$ of order $k$ corresponds a formally integrable finite form $P_{k} \subset Q_{k}$ and its prolongations $P_{k+r} \subset Q_{k+r}$. We may pose the integrability problem for $R_{k}$, as we did for $\Gamma$, in terms of the prolongations of $P_{k}$; its solvability depends only on $R_{k}$ and not on the choice of $\boldsymbol{P}_{\boldsymbol{k}}$. In particular, the integrability problem for the pseudogroup $\Gamma$ depends only on the Lie equation for its infinitesimal transformations. In [12], we associate to a Lie equation $R_{k} \subset J_{k}(T)$ a nonlinear Spencer cohomology $\tilde{H}^{1}\left(R_{k}\right)_{x}$, for each $x \in X$, which is a set with distinguished element 0 ; we say that it vanishes if it is equal to 0 . The vanishing of $H^{1}\left(R_{k}\right)_{x}$ expresses that the integrability problem for $R_{k}$ is solvable at $x \in X$.

3. Nonlinear cohomology of transitive Lie algebras. Consider the real line $\mathbf{R}$ endowed with the discrete topology and linearly compact topological vector spaces over $\mathbf{R}$, i.e., those which are topological duals of real vector spaces endowed with the discrete topology. A transitive Lie algebra is a topological Lie algebra over $\mathbf{R}$ whose underlying topological vector space is linearly compact and satisfies the descending chain condition on closed ideals. To verify that a linearly compact Lie algebra satisfies this last condition, it suffices to verify that it possesses a neighborhood of 0 containing no ideals other than 0 . This class of Lie algebras was introduced by Guillemin and Sternberg ([15] and [14]).

Let $R_{k} \subset J_{k}(T)$ be a Lie equation on the manifold $X$. For $x \in X$, endow $R_{k+l, x}$ with the discrete topology and $R_{\infty, x}=$ proj $\lim R_{k+l, x}$ with the projective limit topology. Then $R_{\infty, x}$ is a topological Lie algebra and a closed subalgebra of $J_{\infty}(T)_{x}$. If $R_{k}$ is formally transitive, the kernel $R_{\infty, x}^{0}$ of $\pi_{0}$ : $R_{\infty, x} \rightarrow J_{0}(T)_{x}$ contains no ideals of $R_{\infty, x}$ other than 0 and so $R_{\infty, x}$ is a transitive Lie algebra. If $X$ is connected and $x, x^{\prime} \in X$, then $R_{\infty, x}$ and $R_{\infty, x^{\prime}}$ are isomorphic as topological Lie algebras.

In [8], we announced a program to investigate the relationship between Lie equations and transitive Lie algebras in order to show in what way certain properties of a formally transitive analytic Lie equation $R_{k}$ depend only on the transitive Lie algebra $R_{\infty, x}$ of formal solutions of $R_{k}$ at $x \in X$ and to what extent the classical theory of finite-dimensional Lie groups and their Lie algebras can be generalized to Lie equations and transitive Lie algebras. In the papers [9], [10], [11], [12] and [13], we have undertaken our study of the correspondence between Lie equations and transitive Lie algebras. Here we are mainly concerned with the relationship between the nonlinear Spencer cohomology of a Lie equation and the Lie algebra of formal solutions of the equation. bras:

We have the following algebraic realization result for transitive Lie alge-

THeOREM 1 (GuILlemin-STERNBERG [15]). Let $L$ be a transitive Lie algebra. Then there exist a manifold $X$ and $x \in X$ such that $L$ is isomorphic to a closed subalgebra $L^{\prime}$ of $J_{\infty}(T)_{x}$ for which $\pi_{0}: L^{\prime} \rightarrow J_{0}(T)_{x}$ is surjective.

Using the above theorem and existence theorems for analytic nonlinear 
partial differential equations, in [10] we prove:

Theorem 2 (Third Fundamental TheOREM). Let $L$ be a transitive Lie algebra. Then there exists an analytic formally transitive Lie equation $R_{k}$ of order $k$ on an analytic manifold $X$ such that, for $x \in X$, the transitive Lie algebra $R_{\infty, x}$ is isomorphic to $L$.

This analogue of Lie's third fundamental theorem for Lie groups shows that all transitive Lie algebras arise from formally transitive Lie equations. The following theorem of [11] shows that closed ideals of a transitive Lie algebra also correspond to Lie equations.

THEOREM 3. Let $R_{k}$ be a formally transitive Lie equation of order $k$ on a simply connected manifold $X$. Let $x \in X$ and I be a closed ideal of $R_{\infty, x}$. Then there exist an integer $k_{1} \geqslant k$ and a unique Lie equation $N_{k_{1}} \subset R_{k_{1}}$ of order $k_{1}$ on $X$ such that

$$
N_{\infty, x}=I \text { and }\left[R_{k_{1}+1}, N_{k_{1}+1}\right] \subset N_{k_{1}}
$$

We say that two cohomologies are isomorphic if they are connected by a bijective mapping sending 0 into 0 , and we shall identify two cohomologies if there is an isomorphism of cohomology between them. We now associate to a transitive Lie algebra $L$ a nonlinear Spencer cohomology $\tilde{H}^{1}(L)$ and to a closed ideal $I$ of $L$ a nonlinear Spencer cohomology $\tilde{H}^{1}(L, I)$. According to Theorems 2 and 3 , there exist a formally transitive analytic Lie equation $R_{k} \subset J_{k}(T)$ on an analytic manifold $X$, a point $x \in X$, a Lie equation $N_{k_{1}} \subset R_{k_{1}}$ of order $k_{1} \geqslant k$ such that

$$
\left[R_{k_{1}+1}, N_{k_{1}+1}\right] \subset N_{k_{1}}
$$

and $\left(R_{\infty, x}, N_{\infty, x}\right)$ and $(L, I)$ are isomorphic as pairs of topological Lie algebras. We define

$$
\tilde{H}^{1}(L)=\tilde{H}^{1}\left(R_{k}\right)_{x}, \quad \tilde{H}^{1}(L, I)=\tilde{H}^{1}\left(N_{k_{1}}\right)_{x} .
$$

We have $\tilde{H}^{1}(L, L)=\tilde{H}^{1}(L)$. The next two theorems are proved in $\S 10$ of [12].

THEOREM 4. The nonlinear Spencer cohomology $\tilde{H}^{1}(L, I)$ of a closed ideal I of a transitive Lie algebra $L$ is well defined and depends only on the isomorphism class of $(L, I)$ as a pair of topological Lie algebras.

This last result says that the cohomology $\tilde{H}^{1}\left(R_{k}\right)_{x}$ of an analytic formally transitive Lie equation $R_{k} \subset J_{k}(T)$ on a connected manifold $X$ is independent of the point $x \in X$ and depends only on the transitive Lie algebra $R_{\infty, x^{*}}$

THEOREM 5. Let $\phi: L \rightarrow L^{\prime \prime}$ be a continuous epimorphism of transitive Lie algebras and let $I$ be the closed ideal of $L$ which is the kernel of $\phi$. If $\tilde{H}^{1}(L, I)=0$ and $\tilde{H}^{1}\left(L^{\prime \prime}\right)=0$, then $\tilde{H}^{1}(L)=0$.

These two theorems are comparison results for the existence of solutions of nonlinear partial differential equations, which in general are defined on manifolds of different dimensions. In particular, if $R_{k}$ and $R_{p}^{\#}$ are formally transitive analytic Lie equations on connected analytic manifolds $X$ and $Y$ 
respectively and if $x \in X$ and $y \in Y$ are points such that the transitive Lie algebras $R_{\infty, x}$ and $R_{\infty, y}^{\#}$ are isomorphic, then the solvability of the integrability problem for $R_{k}$ is equivalent to that of the integrability problem for $R_{p}^{\#}$.

The crucial part of the proofs of Theorems 4 and 5 is provided by Theorem 9.1 of [12], which we now describe. If a pseudogroup $\Gamma$ acting on a manifold $X$ preserves a fibration $\rho: X \rightarrow Y$, then under certain assumptions on $\Gamma$ (which hold in particular when $\Gamma$ is transitive) the theorem of KuranishiRodrigues [18] (see also [11] and [13]) asserts that the set of diffeomorphisms of $Y$ which are projections of transformations belonging to $\Gamma$ is a Lie pseudogroup; the Lie equation $R_{k}$ on $X$ corresponding to $\Gamma$ determines in this way a Lie equation $R_{k_{1}}^{\prime \prime}$ on $Y$. Theorem 9.1 of [12] establishes the key fact that under these conditions on $\Gamma$ the nonlinear cohomology of $R_{k}$ is isomorphic to that defined in terms of $\rho$-projectable sections; the solvability of equation (8) is equivalent to that of the same equation when $u$ is $\rho$-projectable as defined in $\$ 4$ of [12]. This theorem determines a mapping

$$
\rho: \tilde{H}^{1}\left(R_{k}\right)_{x} \rightarrow \tilde{H}^{1}\left(R_{k_{1}}^{\prime \prime}\right)_{\rho(x)},
$$

for $x \in X$, which enables us to compare the integrability problems for $R_{k}$ and $R_{k_{1}}^{\prime \prime}$ and to construct the exact sequences of nonlinear cohomology of $\$ 9$ of [12], which are the tools used in proving Theorems 4 and 5.

Since the proof of Theorem 9.1 of [12] is quite long and complicated and requires $\$ 4, \S 6$ and $\S 8$ of [12], we give here only a very brief outline of the proof decomposed into three steps.

(A) First, one uses the vanishing of the nonlinear Spencer cohomology of a certain multifoliate Lie equation, proved in $\$ 8$ of [12] by means of Frobenius' theorem, to replace the section $u$ of equation (8) satisfying $\mathscr{Q}_{1} u=0$ by one verifying an additional condition with respect to $\rho$. In the case of a formally transitive Lie equation, the vanishing result used is equivalent to the solvability of the integrability problem for $G$-structures, with $G=\mathrm{Gl}(n, k ; \mathbf{R})$.

(B) The main step consists in setting up and solving a nonlinear partial differential equation along the fibers of $\rho$

$$
\mathfrak{D}_{X / Y} \phi=w,
$$

where $w$ is obtained from the $u$ given by step (A). Because $w$ satisfies an integrability condition $\mathscr{D}_{1, X / Y} w=0$, according to $\$ 4$ of [12] and Frobenius' theorem, we find a solution $\phi$ of this equation.

(C) Finally, using $\phi$ we modify the $u$ given by step (A) so that it becomes essentially $\rho$-projectable.

This theorem was stated for transitive pseudogroups in a different framework by Pollack [21] and Molino [20]. These authors carried out the analogue of step (A). However the result does not follow directly from (A); the following steps (B) and (C) are in fact the essential ones-a point which apparently had been overlooked.

4. The Jordan-Hölder decomposition and the integrability problem. The systematic study of transitive Lie algebras resulted in the fundamental paper [14] of Guillemin in which a Jordan-Hölder decomposition is constructed for a transitive Lie algebra. This decomposition is an outgrowth of a program 
outlined by Guillemin in the introduction of [14] which was motivated by the integrability problem.

THEOREM 6 (GUILLEMIN [14]). Let $L$ be a transitive Lie algebra. Then there is a nested sequence

$$
L=I_{0} \supset I_{1} \supset I_{2} \supset \cdots \supset I_{k}=0
$$

of closed ideals of $L$ such that, for each $j$, where $0 \leqslant j \leqslant k-1$, either $I_{j} / I_{j+1}$ is abelian or there are no closed ideals of $L$ properly contained between $I_{j}$ and $\boldsymbol{I}_{\boldsymbol{j}+\boldsymbol{1}}$.

A sequence (9) satisfying the conditions described in the theorem is a Jordan-Hölder sequence for $L$. Because of Theorem 5 , one can show that the nonlinear Spencer cohomology $\tilde{H}^{1}(L)$ of a transitive Lie algebra $L$ vanishes by proving that the cohomologies $\tilde{H}^{1}\left(L / I_{j+1}, I_{j} / I_{j+1}\right)$ obtained from the Jordan-Hölder sequence (9) for $L$ vanish for $0 \leqslant j \leqslant k-1$. It is therefore essential to compute the nonlinear Spencer cohomology of a closed ideal $I$ of a transitive Lie algebra under the assumption that $I$ is either

(a) abelian or

(b) nonabelian and minimal.

We begin by considering the case of closed abelian ideals of transitive Lie algebras.

THEOREM 7 [13]. Let I be a closed abelian ideal of a transitive Lie algebra $L$. Then there exist a Lie pseudogroup $\Gamma$ on a vector bundle $\rho: E \rightarrow Y$ over a manifold $Y$ whose associated Lie equation $R_{k} \subset J_{k}(T)$ is of order $k$ on $X=E$, vector bundles $F$ and $G$ over $Y$, and formally integrable linear differential operators $P: \mathcal{F} \rightarrow \mathscr{F}$ of order $k$ and $Q: \mathscr{F} \rightarrow \mathcal{G}$ of order $l$ on $Y$ such that:

(i) $\left[R_{m}, R_{m}\right]=0$, for all $m \geqslant k$;

(ii) $R_{\infty, x}$ is isomorphic to I as topological Lie algebras, for all $x \in X$;

(iii) $\Gamma$ consists of all local diffeomorphisms of $X$ of the form

$$
x \mapsto x+s(\rho(x)), \quad x \in X,
$$

where $s$ is a solution of the homogeneous equation $P s=0$ over an open subset of $\boldsymbol{Y}$;

(iv) the differential operator $Q$ is a compatibility condition for $P$, i.e., the sequence (4) is formally exact;

(v) the cohomologies $\tilde{H}^{1}(L, I), \tilde{H}^{1}\left(R_{k}\right)_{x}, H^{1}\left(R_{k}\right)_{m, x}$, and the cohomology of the sequence (4) at $\rho(x)$ are isomorphic for all $m \geqslant k+l$ and $x \in X$.

Therefore the nonlinear Spencer cohomology of the ideal $I$ of $L$ vanishes if and only if the differential operator $P$ is locally solvable.

Let $Y$ be a Lie group and $E, F$ be $Y$-bundles over $Y$; let $P: \mathcal{E} \rightarrow \mathscr{F}$ be an invariant differential operator on $Y$. The pseudogroup $\Gamma$ defined in assertion (iii) of Theorem 7, where $X=E$ and $\rho: E \rightarrow Y$ is the natural projection, corresponds to a Lie equation $R_{k} \subset J_{k}(T)$ on $X$. The nonlinear cohomology $\tilde{H}^{1}\left(R_{k}\right)_{x}$ at $x \in X$ is isomorphic to the cohomology of the sequence (4) at $\rho(x)$, where $Q$ is a compatibility condition for $P$. Let $g$ denote the Lie algebra of $Y$ and $M_{y}$ the $g$-module of formal solutions of $P$ at $y \in Y$. If $\pi_{0}$ : 
$M_{y} \rightarrow J_{0}(E)_{y}$ is surjective for $y \in Y$, according to [13] the pseudogroup $\Gamma^{\prime}$ on $X$ generated by the action of the elements of $Y$ on $E$ and by $\Gamma$ is transitive and corresponds to a formally transitive Lie equation $R_{k_{0}}^{\prime} \subset J_{k_{0}}(T)$, with $k_{0} \geqslant k$, satisfying:

(i) $\left[R_{k_{0}+1}^{\prime}, R_{k_{0}+1}\right] \subset R_{k_{0}}$;

(ii) $R_{\infty, x}$ is a closed abelian ideal of $R_{\infty, x}^{\prime}$, for $x \in X$;

(iii) if $L_{y}$ is the semidirect product of $g$ and $M_{y}$, with $y \in Y$, there is an isomorphism of pairs of Lie algebras

$$
\left(R_{\infty, x}^{\prime}, R_{\infty, x}\right) \rightarrow\left(L_{\rho(x)}, M_{\rho(x)}\right),
$$

for $x \in X$;

(iv) the nonlinear cohomology of $R_{k_{0}}^{\prime}$ at $x \in X$ is isomorphic to $\tilde{H}^{1}\left(R_{k}\right)_{x}$ and thus vanishes if and only if $P$ is locally solvable at $\rho(x)$.

If the differential operator $P$ is not locally solvable, the integrability problem is not solvable for the Lie equation $R_{k_{0}}^{\prime}$.

We now point out how the counterexample to the solvability of the integrability problem of Guillemin and Sternberg [16] arises in this way. Let $Y$ be the Lie group SU(2) and let $\left\{\eta_{1}, \eta_{2}, \eta_{3}\right\}$ be a basis for the Lie algebra of left-invariant vector fields on $Y$ such that the relations

$$
\left[\eta_{i}, \eta_{j}\right]=\eta_{l}
$$

hold for all cyclic permutations $(i, j, l)$ of $(1,2,3)$. Let $\theta_{Y}$ denote the sheaf of complex-valued functions on $Y$. Under the standard identification of $Y$ with the three-dimensional sphere $S^{3}$ imbedded in $C^{2}$, the differential operator $\bar{\partial}_{b}$ : $\theta_{Y} \rightarrow \theta_{Y}$ determined by the complex vector field $\eta_{1}+\sqrt{-1} \eta_{2}$ on $Y$ coincides essentially with the tangential Cauchy-Riemann operator on the real hypersurface $S^{3}$ of $\mathbf{C}^{2}$, which is the locally nonsolvable operator of H. Lewy. The example of Guillemin and Sternberg [16] is the transitive pseudogroup $\Gamma^{\prime}$ obtained by the above procedure from the differential operator $\bar{\partial}_{b}$ on $Y$. The space $H$ of all formal solutions at the identity element of $S U(2)$ of the equation $\bar{\partial}_{b} u=0$ is a module over the Lie algebra $g$ of $\mathrm{SU}(2)$; we denote by $L$ the semidirect product of $g$ and $H$. Then $L$ possesses a natural structure of transitive Lie algebra such that $H$ is identified with a closed abelian ideal $I$ of $L$. Then

$$
L \supset I \supset 0
$$

is a Jordan-Hölder sequence for $L$, with $L / I$ isomorphic to the simple Lie algebra $g$. Moreover, by the above discussion we have

$$
\tilde{H}^{1}(L) \simeq \tilde{H}^{1}(L, I) \neq 0 .
$$

We next consider a nonabelian minimal closed ideal $I$ of a transitive Lie algebra $L$. According to [14], $I$ possesses a unique maximal closed ideal $J$ of I. Then $R=I / J$ is a simple transitive Lie algebra, i.e., it possesses no nontrivial ideals. Moreover, the commutator ring $K_{R}$ of $R$ (i.e., the algebra of all linear mappings $R \rightarrow R$ which commute with all the mappings ad $\xi$ : $R \rightarrow R$ with $\xi \in R$ ) is equal to $R$ or $C$, and we say that $I$ is of real or complex type according to whether $K_{R}$ is $\mathbf{R}$ or $\mathbf{C}$. The infinite simple transitive Lie algebras are classified. 
The structure theorem of Guillemin [14] gives us an isomorphism of Lie algebras

$$
\phi: I \rightarrow R \hat{\otimes}_{K_{R}} F,
$$

where $F$ is the power series ring $K_{R}\left[\left[x_{1}, \ldots, x_{p}\right]\right]$ in $p$ indeterminates $x_{1}, \ldots, x_{p}$ over $K_{R}$ endowed with the Krull topology and where the tensor product is a completion of the usual algebraic tensor product.

If $K_{R}=\mathbf{R}$, by a detailed analysis of Guillemin's work, following an outline given in $\$ 13$ of [12], Conn [5] shows that the Lie algebra $\operatorname{Der}\left(R \hat{\otimes}_{\mathrm{R}} F\right)$ of continuous derivations of $R \hat{\otimes}_{\mathrm{R}} F$ has a natural structure of transitive Lie algebra, that $R \hat{\otimes}_{\mathrm{R}} F$ can be identified with a closed ideal of this algebra, and that the mapping $\phi$ is the restriction of a continuous homomorphism of transitive Lie algebras

$$
\phi: L \rightarrow \operatorname{Der}\left(R \hat{\otimes}_{\mathrm{R}} F\right) .
$$

Moreover, this homomorphism $\phi$ induces an isomorphism of cohomology

$$
\tilde{H}^{1}(L, I) \stackrel{\sim}{\rightarrow} \tilde{H}^{1}\left(\operatorname{Der}\left(R \hat{\otimes}_{\mathrm{R}} F\right), R \hat{\otimes}_{\mathrm{R}} F\right) .
$$

The fact that the cohomology $\tilde{H}^{1}\left(\operatorname{Der}\left(R \hat{\otimes}_{\mathrm{R}} F\right), R \hat{\otimes}_{\mathrm{R}} F\right)$ vanishes follows from the classification of the infinite simple transitive Lie algebras and Frobenius' or Darboux's theorem with parameters and will be established in a future publication. The pseudogroup $\Gamma$ corresponding to the closed ideal $R \hat{\otimes}_{R} F$ of $\operatorname{Der}\left(R \hat{\otimes}_{R} F\right)$ can be described as follows. Let $\Gamma_{0}$ be a transitive Lie pseudogroup acting on a manifold $X$ whose associated transitive Lie algebras are isomorphic to $R$ and let $Y$ be a manifold of dimension $p$. Then the pseudogroup $\Gamma$ consists of all local diffeomorphisms of $X \times Y$ which leave each fiber of the projection of $X \times Y$ onto $Y$ invariant and whose restrictions to each fiber belong to $\Gamma_{0}$. We thus obtain:

THEOREM 8. Let $L$ be a transitive Lie algebra and I a nonabelian minimal closed ideal of $L$. If $I$ is of real type, then $\tilde{H}^{1}(L, I)=0$.

Finally, we examine the case when $I$ is of complex type. Conn [5] associates to such an ideal a real-analytic submanifold $Y$ of $\mathbf{C}^{p}$ defined in a neighborhood of $0 \in C^{p}$. The Levi form of $I$ is the Levi form of $Y$ at 0 defined in terms of the pseudo-complex structure on $Y$ induced by the complex structure of $\mathbf{C}^{p}$. By a formal analogue of the $\mathrm{H}$. Lewy extension phenomenon, the ring $H$ of all formal solutions at 0 of the induced Cauchy-Riemann operator $\bar{\partial}_{b}$ : $\theta_{Y} \rightarrow \theta_{Y}$ is isomorphic to the ring $F$, considered as the space of all formal holomorphic functions on $\mathbf{C}^{p}$ at 0 . Thus $I$ is isomorphic to the Lie algebra $R \hat{\otimes}_{\mathrm{C}} H$. The integrability problem for $I$ is equivalent to that for the pseudogroup $\Gamma$ which we now describe. Let $\Gamma_{0}$ be a transitive Lie pseudogroup of holomorphic transformations acting on a complex manifold $X$ whose associated transitive Lie algebras are isomorphic to $R$. Then the pseudogroup $\Gamma$ is generated by all local diffeomorphisms of $X \times Y$ which are of the form $(x, y) \mapsto(\varphi(x, y), y)$, which leave each fiber of the projection of $X \times Y$ onto $Y$ invariant, whose restrictions to each fiber belong to $\Gamma_{0}$ and which moreover satisfy the differential equation $\bar{\partial}_{b} \varphi=0$.

If the Levi form of $I$ vanishes, then $Y$ is a family of complex manifolds and 
the operator $\overline{\partial_{b}}$ is simply the operator $\bar{\partial}$ acting along the fibers of this family. The fact that the integrability problem for the pseudogroup $\Gamma$ is solvable follows from the Newlander-Nirenberg theorem with parameters and will be proved in a future publication. As a consequence, we obtain:

THEOREM 9. Let $L$ be a transitive Lie algebra and I a nonabelian minimal closed ideal of $L$. If $I$ is of complex type and its Levi form vanishes, then $\tilde{H}^{1}(L, I)=0$.

On the other hand, if the Levi form of $I$ does not vanish, the integrability problem for $\Gamma$ need not be solvable, as shown by the recent examples of Conn [4]. We now construct one of these examples. Let $Y$ be the Lie group SU(2) with its pseudo-complex structure obtained from the imbedding of the sphere $S^{3}$ into $C^{2}$. If $\Gamma_{0}$ is the pseudogroup of all local biholomorphic transformations of $X=\mathbf{C}$, the integrability problem is not solvable for the pseudogroup $\Gamma$ defined above in terms of $\Gamma_{0}$ and SU(2). In fact, in [4] it is shown that the integrability problem is not solvable for the transitive pseudogroup $\Gamma^{\prime}$ acting on $\mathrm{SU}(2) \times \mathbf{C}$ generated by $\Gamma$ and the left-translations of $\mathrm{SU}(2)$. If $L$ is the transitive Lie algebra associated to $\Gamma^{\prime}$ at a point of $X \times Y$, the closed ideal $I$ of $L$ corresponding to the sub-pseudogroup $\Gamma$ of $\Gamma^{\prime}$ is nonabelian, minimal, and of complex type, and

$$
\tilde{H}^{1}(L) \simeq \tilde{H}^{1}(L, I) \neq 0 .
$$

Moreover

$$
L \supset I \supset 0
$$

is the unique Jordan-Hölder sequence for $L$ and all of its quotients are nonabelian.

Now let $\Gamma$ be a pseudogroup acting on $\mathbf{R}^{n}$ containing all translations. Let (9) be a Jordan-Hölder sequence for the transitive Lie algebra $L$ associated to $\Gamma$ and $0<j<k-1$. If the closed ideal $I_{j} / I_{j+1}$ of $L / I_{j+1}$ is abelian, in [12] we show that the linear differential operator $\boldsymbol{P}$ given by Theorem 7 has constant coefficients in an appropriate coordinate system; the theorem of Ehrenpreis-Malgrange asserts that $P$ is locally solvable and thus $\tilde{H}^{1}\left(L / I_{j+1}\right.$, $\left.I_{j} / I_{j+1}\right)=0$. On the other hand, if the closed ideal $I_{j} / I_{j+1}$ of $L / I_{j+1}$ is nonabelian and minimal, a result of Guillemin's implies that, if $I_{j} / I_{j+1}$ is of complex type, its Levi form vanishes (see Conn [5]). Therefore by Theorems 8 and 9 , the cohomology $\tilde{H}^{1}\left(L / I_{j+1}, I_{j} / I_{j+1}\right)$ vanishes. By repeated applications of Theorem 5, we obtain the vanishing of $\tilde{H}^{1}(L)$ and

THEOREM 10. The integrability problem is solvable for pseudogroups acting on $\mathbf{R}^{n}$ containing all translations.

In particular, the integrability problem is solvable for "flat" pseudogroups (see [15]). Since the pseudogroups $\Gamma_{G}$ of $\$ 1$ acting on $\mathbf{R}^{n}$ contain all translations, Theorem 10 implies that the integrability problem for $G$-structures is solvable.

We restate Theorem 10 in terms of Lie equations or transitive Lie algebras:

THEOREM 11. Let $R_{k} \subset J_{k}(T)$ be a formally transitive Lie equation on a connected manifold $X$. If there is a point $x \in X$ and an abelian subalgebra $A$ of 
$R_{\infty, x}$ such that

$$
R_{\infty, x}=R_{\infty, x}^{0} \oplus A,
$$

then $\tilde{H}^{1}\left(R_{k}\right)_{a}=0$, for all $a \in X$.

TheOREM 12. Let $L$ be a transitive Lie algebra. If there exist an open subalgebra $L^{0}$ of $L$ containing no ideals of $L$ other than 0 and an abelian subalgebra $A$ of $L$ such that

then $\tilde{H}^{1}(L)=0$.

$$
L=L^{0} \oplus A,
$$

\section{REFERENCES}

1. C. Buttin and P. Molino, Théorème général d'équivalence pour les pseudogroupes de Lie plats transitifs, J. Differential Geometry 9 (1972), 347-354.

2. E. Cartan, Sur la structure des groupes infinis de transformations, Ann. Sci. École Norm. Sup. 21 (1904), 153-206; 22 (1905), 219-308; Oeuvres complètes: II, vol. 2, Groupes infinis, systèmes différentiels, théories d'équivalence, Gauthier-Villars, Paris, 1953, pp. 571-714.

3. _ La structure des groupes infinis, Oeuvres complètes: II, vol. 2, Gauthier-Villars, Paris, 1953, pp. 1335-1384.

4. J. F. Conn, A new class of counterexamples to the integrability problem, Proc. Nat. Acad. Sci. U.S.A. 74 (1977), 2655-2658.

5. _ Non-abelian minimal closed ideals of transitive Lie algebras, Ph.D. thesis, Princeton Univ., Princeton, N. J., 1978.

6. H. Goldschmidt, Existence theorems for analytic linear partial differential equations, Ann. of Math. (2) 86 (1967), 246-270.

7. Ann. Sci. Ecole Norm. Sup. (4) 1 (1968), 417-444.

8. _ On the Spencer cohomology of a Lie equation, Proc. Sympos. Pure Math., vol. 23 Amer. Math. Soc., Providence, R. I., 1973, pp. 379-385.

9. __ Sur la structure des équations de Lie: I. Le troisième théorème fondamental, J. Differential Geometry 6 (1972), 357-373.

10. __ Sur la structure des équations de Lie: II. Équations formellement transitives, J. Differential Geometry 7 (1972), 67-95.

11. __ Sur la structure des équations de Lie: III. La cohomologie de Spencer, J. Differential Geometry 11 (1976), 167-223.

12. H. Goldschmidt and D. Spencer, On the non-linear cohomology of Lie equations. I, II, Acta Math. 136 (1976), 103-239.

13. __ On the non-linear cohomology of Lie equations. III, IV, J. Differential Geometry 13 (1978).

14. V. W. Guillemin, A Jordan-Hölder decomposition for a certain class of infinite dimensional Lie algebras, J. Differential Geometry 2 (1968), 313-345.

15. V. W. Guillemin and S. Sternberg, An algebraic model of transitive differential geometry, Bull. Amer. Math. Soc. 70 (1964), 16-47.

16. The Lewy counterexample and the local equivalence problem for G-structures, J. Differential Geometry 1 (1967), 127-131.

17. A. Kumpera and D. Spencer, Lie equations. Vol. I. General theory, Ann. of Math. Studies No. 73, Princeton Univ. Press and Univ. of Tokyo Press, 1972.

18. M. Kuranishi and A. A. M. Rodrigues, Quotients of pseudo groups by invariant fiberings, Nagoya Math. J. 24 (1964), 109-128.

19. B. Malgrange, Equations de Lie. I, II, J. Differential Geometry 6 (1972), 503-522; 7 (1972), 117-141. 
20. P. Molino, Théorie des G-structures: Le problème d'équivalence, Lecture Notes in Math., vol. 588, Springer-Verlag, Berlin and New York, 1977.

21. A. S. Pollack, The integrability problem for pseudogroup structures, J. Differential Geometry 9 (1974), 355-390.

22. D. C. Spencer, Deformation of structures on manifolds defined by transitive, continuous pseudogroups. I, II, Ann. of Math. (2) 76 (1962), 306-445.

Department of Mathematics, Rutgers University, New Brunswick, New Jersey 08903 